\title{
CONTROL OF COMBUSTION-INSTABILITIES THROUGH VARIOUS PASSIVE DEVICES
}

\author{
Abdelkader Frendi \\ University of Alabama in Huntsville \\ Huntsville, AL 35899 \\ Tom Nesman and Francisco Canabal \\ NASA Marshall Space Flight Center \\ Huntsville, AL 35812
}

\begin{abstract}
Results of a computational study on the effectiveness of various passive devices for the control of combustion instabilities are presented. An axi-symmetric combustion chamber is considered. The passive control devices investigated are, baffles, Helmholtz resonators and quarter-waves. The results show that a Helmholtz resonator with a smooth orifice achieves the best control results, while a baffle is the least effective for the frequency tested. At high sound pressure levels, the Helmholtz resonator is less effective. It is also found that for a quarter wave, the smoothness of the orifice has the opposite effect than the Helmholtz resonator, i.e. results in less control.
\end{abstract}

\section{Introduction}

It is well known that under some operating conditions, rocket engines (using solid or liquid fuels) exhibit unstable modes of operation that can lead to engine malfunction and shutdown. The sources of these instabilities are diverse and are dependent on fuel, chamber geometry and various upstream sources such as pumps, valves and injection mechanism. It is believed that combustion-acoustic instabilities occur when the acoustic energy increase due to the unsteady heat release of the flame is greater than the losses of acoustic energy from the system $[1,2]$. 
Giammar and Putnam [3] performed a comprehensive study of noise generated by gas-fired industrial burners and made several key observations; flow noise was sometimes more intense than combustion roar, which tended to have a characteristic frequency spectrum. Turbulence was amplified by the flame. The noise power varied directly with combustion intensity and also with the product of pressure drop and heat release rate. Karchmer [4] correiated the noise emitted Ifrom a turbofan jei engine with that in the combustion chamber. This is important, since it quantified how much of the noise from an engine originates in the combustor.

A physical interpretation of the interchange of energy between sound waves and unsteady heat release rates was given by Rayleigh [5] for inviscid, linear perturbations. Bloxidge et al [6] extended Rayleigh's criterion to describe the interaction of unsteady combustion with one-dimensional acoustic waves in a duct. Solutions to the mass, momentum and energy conservation equations in the pre- and post-flame zones were matched by making several assumptions about the combustion process. They concluded that changes in boundary conditions affect the energy balance of acoustic waves in the combustor. Abouseif at al [7] also solved the one-dimensional flow equations, but they used a one-step reaction to evaluate the unsteady heat release rate by relating it to temperature and velocity perturbations. Their analysis showed that oscillations arise from coupling between entropy waves produced at the flame and pressure waves originating from the nozzle. Yang and Culick [8] assumed a thin flame sheet, which is distorted by velocity and pressure oscillations. Conservation equations were expressed in integral form and solutions for the acoustic wave equations and complex frequencies were obtained. The imaginary part of the frequency indicated stability regions of the flame.

Activation energy asymptotics together with a one-step reaction were used by McIntosh [9] to study the effects of acoustic forcing and feedback on unsteady, onedimensional flames. He found that the flame stability was altered by the upstream acoustic feedback. Shyy et al [10] used a high-accuracy TVD scheme to simulate unsteady, one-dimensional longitudinal, combustion instabilities. However, numerical diffusion was not completely eliminated. Recently, Prasad [11] investigated numerically the interactions of pressure perturbations with premixed flames. He used complex chemistry to study responses of pressure perturbations in one-dimensional combustors. 
His results indicated that reflected and transmitted waves differed significantly from incident waves.

In the present paper, an extension of the work performed by Frendi [12] is carriedout. In particular, an attempt is made at understanding the onset of combustion instabilities as well as the use of passive devices to control them. Following the

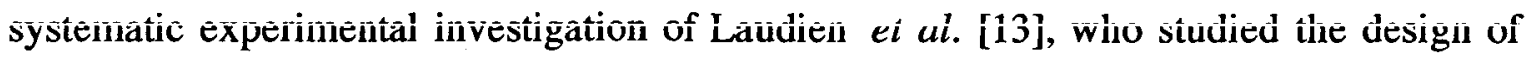
acoustic cavities, a numerical investigation is performed. The remainder of the paper is organized as follows; a brief description of the mathematical model is given in the next section followed by the numerical techniques used to solve it. Details of the results are then discussed followed by the concluding remarks.

\section{Mathematical Model}

The model used to describe this problem is based on the nonlinear Euler equations, which can be written as

$$
\frac{\partial \mathbf{U}}{\partial t}+\frac{\partial \mathbf{F}_{i}}{\partial x_{i}}=\mathbf{B}
$$

where

$$
\mathbf{U}=\left[\begin{array}{c}
\rho \\
\rho v_{j} \\
\rho E
\end{array}\right] \text { and } \mathbf{F}_{i}=\left[\begin{array}{c}
\rho v_{i} \\
\rho v_{i} v_{j}+p \delta_{i j} \\
\rho v_{i}(E+p / \rho)
\end{array}\right]
$$

and $E=c_{v} T+\gamma_{2} v_{i} v_{i}$. The closure is obtained through the equation of state for an ideal gas, $p=\rho R T$. For a two-dimensional axi-symmetric model the definition of the vector $\boldsymbol{B}$ results from the transformation to cylindrical coordinates. If $x_{1}$ is the axis of symmetry, the vector $\boldsymbol{B}$ can be written as

$$
\mathbf{B}=-\frac{1}{x_{2}}\left[\begin{array}{c}
\rho v_{2} \\
\rho v_{2} v_{1} \\
\rho v_{2} v_{2} \\
\rho v_{2}(E+p / \rho)
\end{array}\right] .
$$




\section{Method of Solution}

The numerical methodology employed to solve Equation (1) can be described as a combination of a temporal discretization scheme, a spatial discretization scheme and, a discontinuity-capturing scheme. In the time discretization scheme the value of $U$ is sought given its value at a previous time by means of a Taylor series expansion. The spatial discretization is done using the Galerkin finite element method in which the

integrated weighted-residual is minimized. To resolve discontinuities in the flow field, a convective flux correction term is devised. A second order accuracy is achieved in time while a second order or higher accuracy is achieved in space. Details of the numerical scheme can be found in $[14,15]$. The boundary conditions used are rigid slip wall along the chamber walls and characteristic boundaries at the inlet (upstream boundary) and exit (downstream boundary). When acoustic disturbances are introduced in the chamber, the inlet pressure is specified as

$$
p=p_{0}(1+\varepsilon \sin (\omega t)) \text { or } \quad p=p_{0}(1+\varepsilon R(t))
$$

with $\varepsilon$ being the excitation amplitude, $\omega=2 \pi f$ the frequency and $R(t)$ a random rumber. In Equation (4), $p_{0}$ is the chamber pressure. The other inlet quantities are specified using the method of characteristics.

\section{Results and Discussions}

The numerical investigation carried-out in this paper follows the detailed experimental investigation performed by Laudien et al. [13]. In particular, acoustic tests are performed in an axi-symmetric combustion chamber having the shape shown on Figure 1. A loudspeaker is placed at the center of the inlet boundary and emits plane harmonic or random acoustic waves. As described in section III, a finite element technique is used. Figure 1 also shows a typical grid used. A denser grid is used near the acoustic source. The conditions in the chamber are those of air at $\mathrm{T}=300 \mathrm{~K}$ and $\mathrm{P}=1$ atm. 


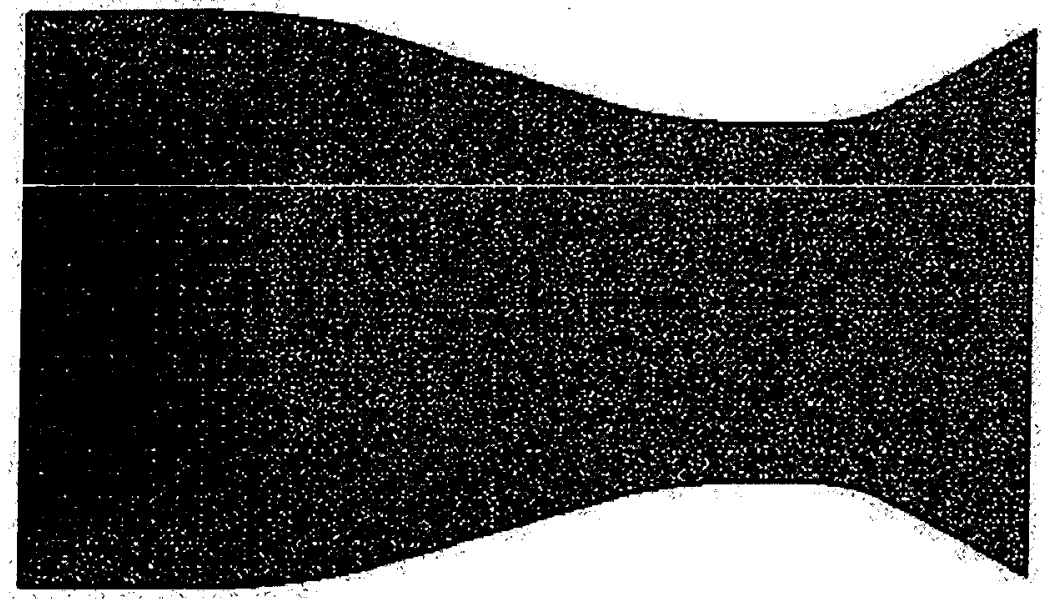

Figure 1: Typical unstructured grid used.

At first, the natural frequencies of the chamber are determined. To this end a random acoustic disturbance is introduced by the loudspeaker and the pressure fluctuations are monitored at several points in the chamber. Figure 2 shows the sound pressure level (SPL) as a function of the frequency at a point along the top chamber wall. Several peaks corresponding to the various chamber modes are obtained. In order $\mathbf{b}$ determine the mode shapes, one can excite the individual modes by introducing a plane, harmonic acoustic wave at the given frequency. Following the determination of the natural frequencies of the chamber, a frequency of $1880 \mathrm{~Hz}$, corresponding to a chamber mode, is selected for extensive tests. The first such test is to obtain the chamber response to a high (i.e. nonlinear) and low (i.e. linear) excitation amplitude. Figures 3(a)-(b) show the time histories of the pressure at an observation point along the top wall. 


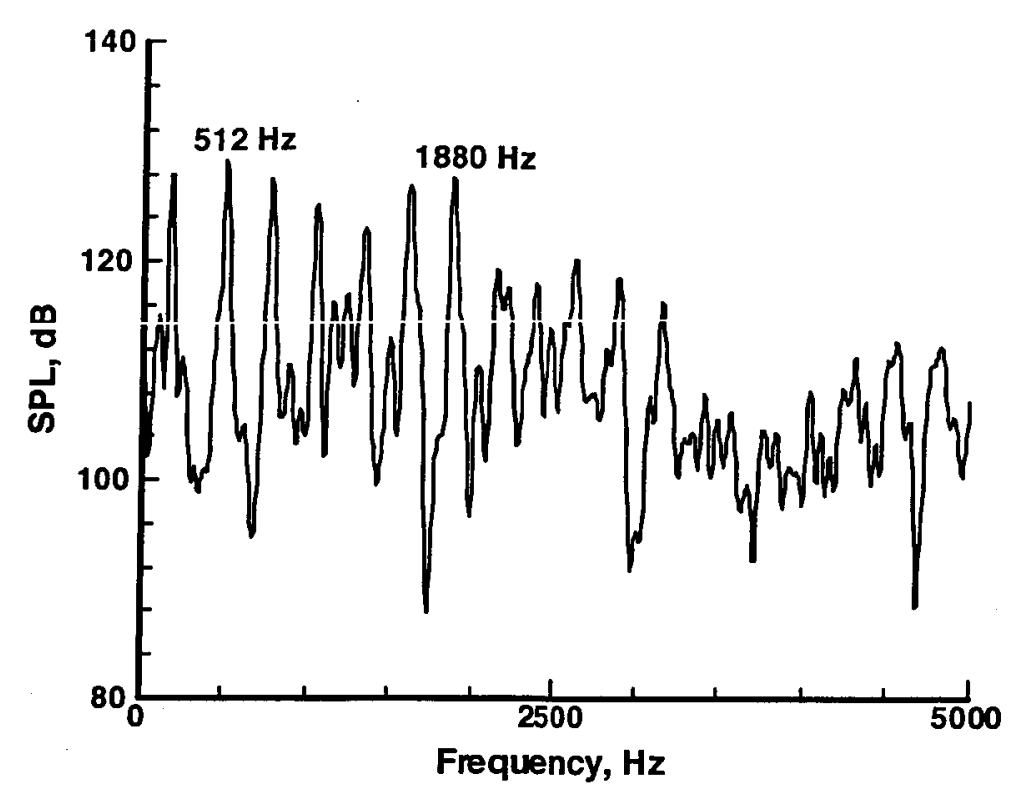

Figure 2: Frequency response of the combustion chamber to a random excitation.

Figure 3(a) shows rapid growth of the pressure oscillations and a slowly varying mean pressure, whereas Figure 3(b) shows a slow growth of the oscillations around a zero mean pressure. The corresponding power spectra are shown on Figs. 4(a)-(b). For the high excitation amplitude case, $\varepsilon=0.5$, strong harmonics are shown to exist along with the fundamental frequency. It is believed that the nonlinear interaction between the fundamental and its harmonics is responsible in part for the rapid growth of the fluctuations. This point is confirmed by Fig. 4(b) which shows weak harmonics and therefore a quasi-linear behavior and weaker growth as shown by Fig. 3(a). 
(a)
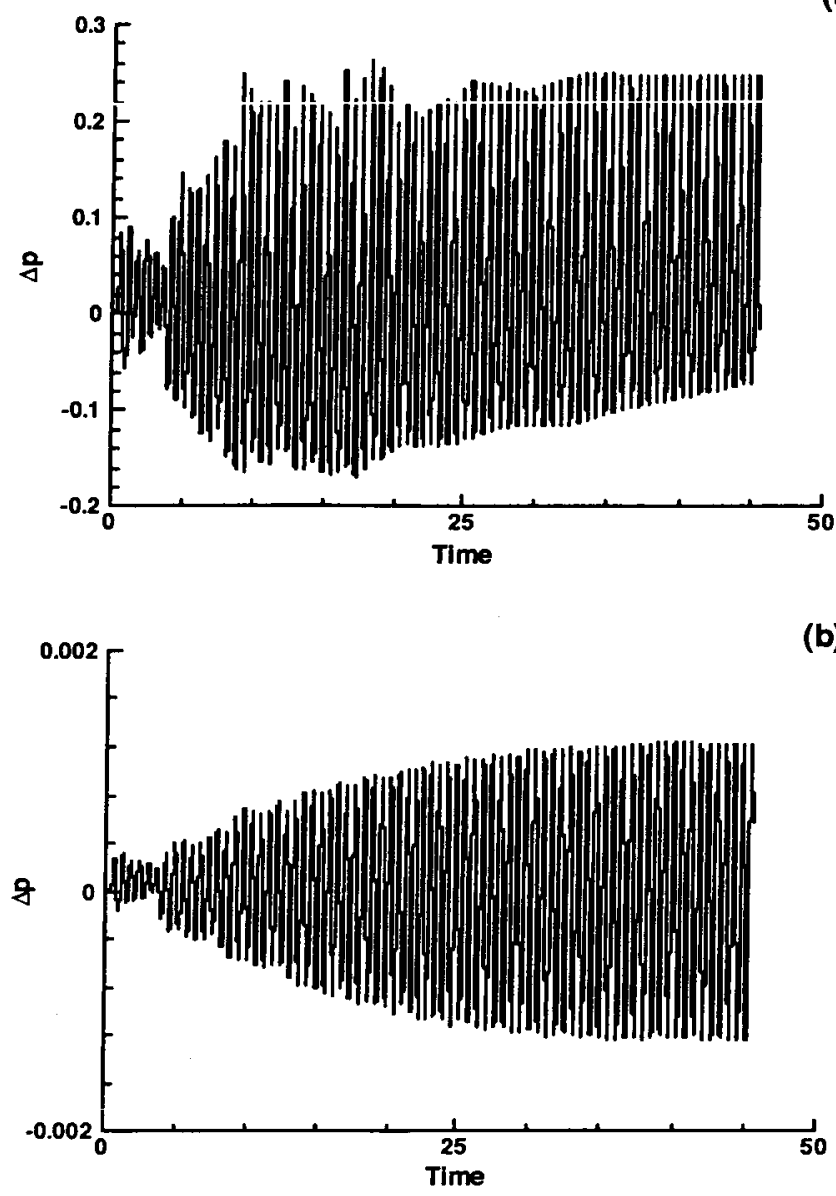

Figure 3 Time history of the pressure at a point along the top wall (a) $\varepsilon=0.5$, (b) $\varepsilon=0.001$ 

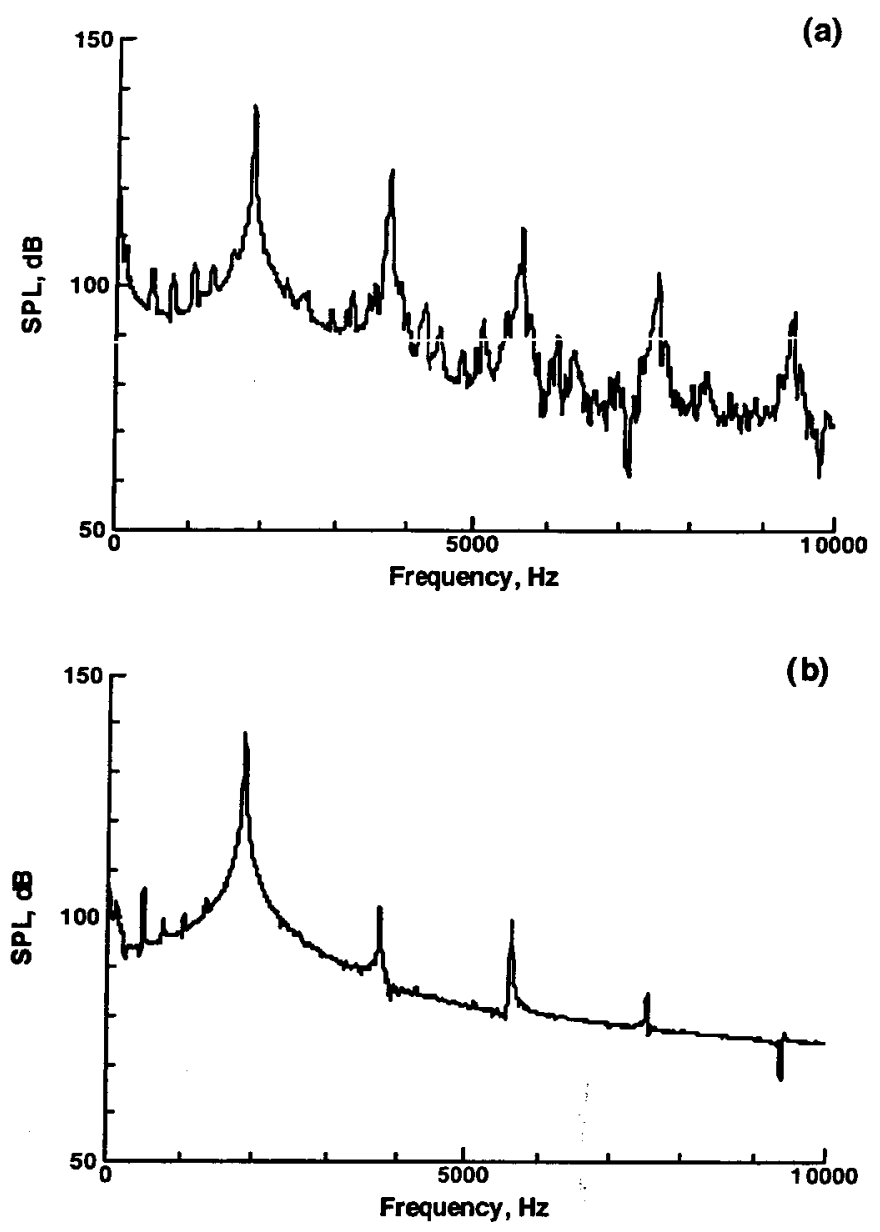

Figure 4: Sound pressure level at a point along the top wall (a) $\varepsilon=0.5$, (b) $\varepsilon=0.001$.

\section{(shard bG "FIQRE $s "$}

In order to assess the level of damping of a given mode by the chamber, the loudspeaker is turned on and then off and the pressure oscillations in the chamber are monitored at a given point. Figure 6 hows the damping level for two different modes 1880 $\mathrm{Hz}$ (Fig. 5(a)) and $512 \mathrm{~Hz}$ (Fig. 5(b)). In addition to the pressure oscillations, a decay curve described by $X e^{-\eta \omega t}$ is also shown, where $\eta$ is the decay factor. The Figure shows that the mode corresponding to $1880 \mathrm{~Hz}$ decays faster than the mode corresponding to $512 \mathrm{~Hz}$. 

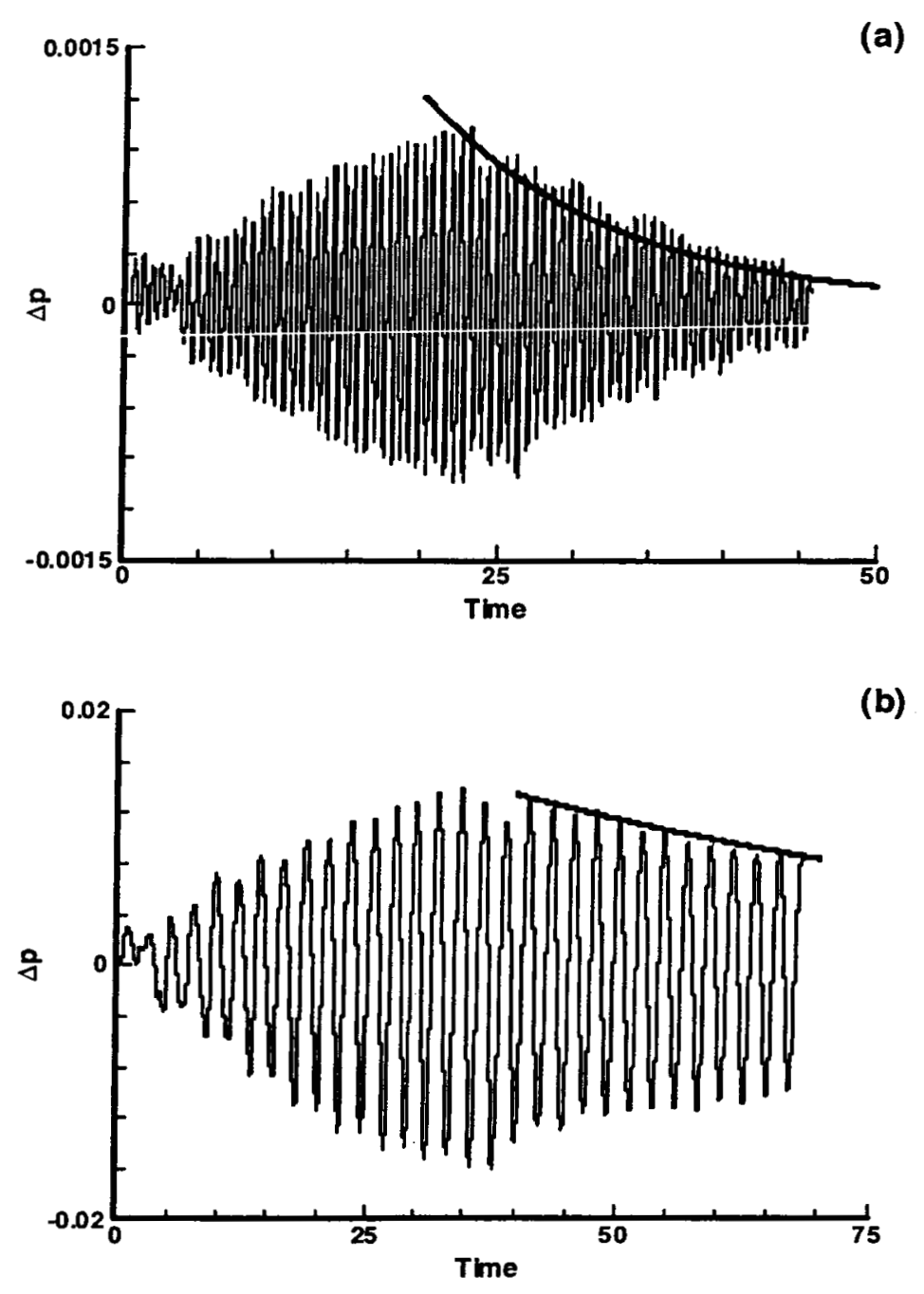

(b)

Figure 5: Decay rate of the pressure oscillations for two different chamber modes corresponding to (a) $1880 \mathrm{~Hz}$ (b) $512 \mathrm{~Hz}$.

\section{IV.1 Baffles}

Following the preliminary studies presented above, the effect of adding a baffle in the radial direction of the inlet boundary, Figure 6 , on the pressure oscillations in the chamber, is investigated. Figure 7 shows the pressure oscillations at the observation point along the top wall obtained with and without the baffle. The Figure shows a significant reduction of the oscillation amplitude of the pressure. The excitation amplitude used is $\varepsilon=0.1$. 
(a)

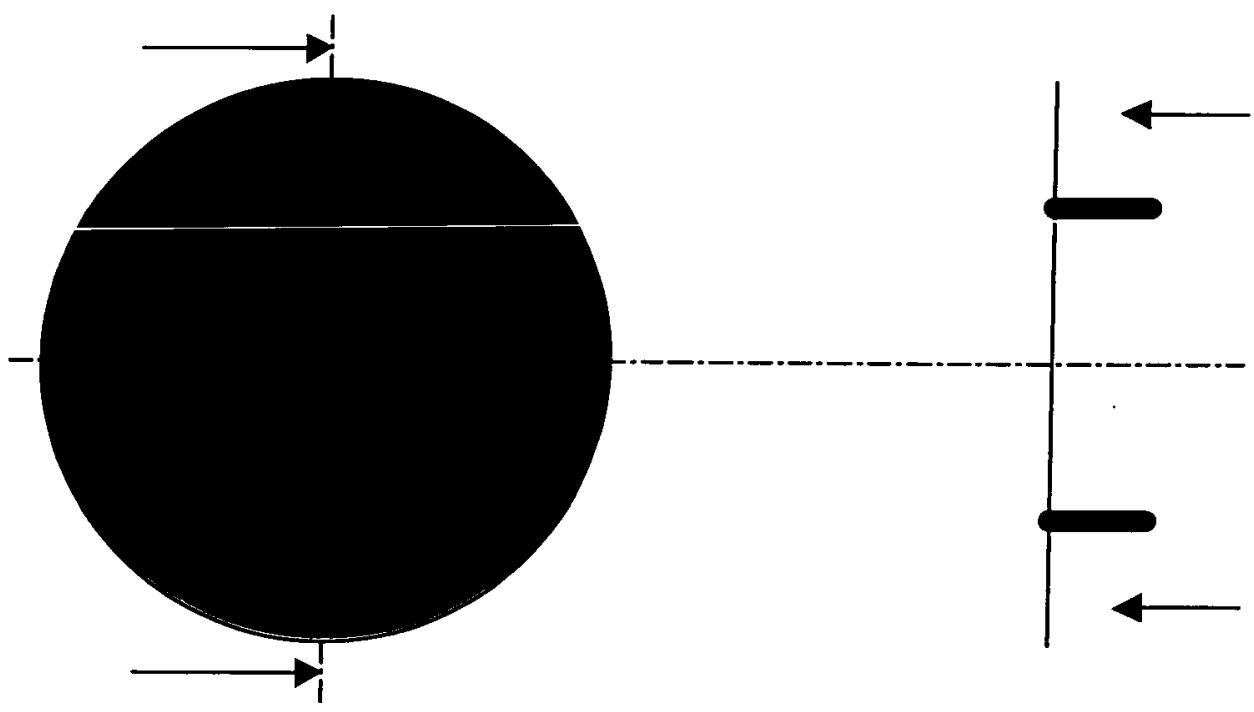

Figure 6: Sketch of the inlet boundary with a circular baffle; (a) front view, (b) side view

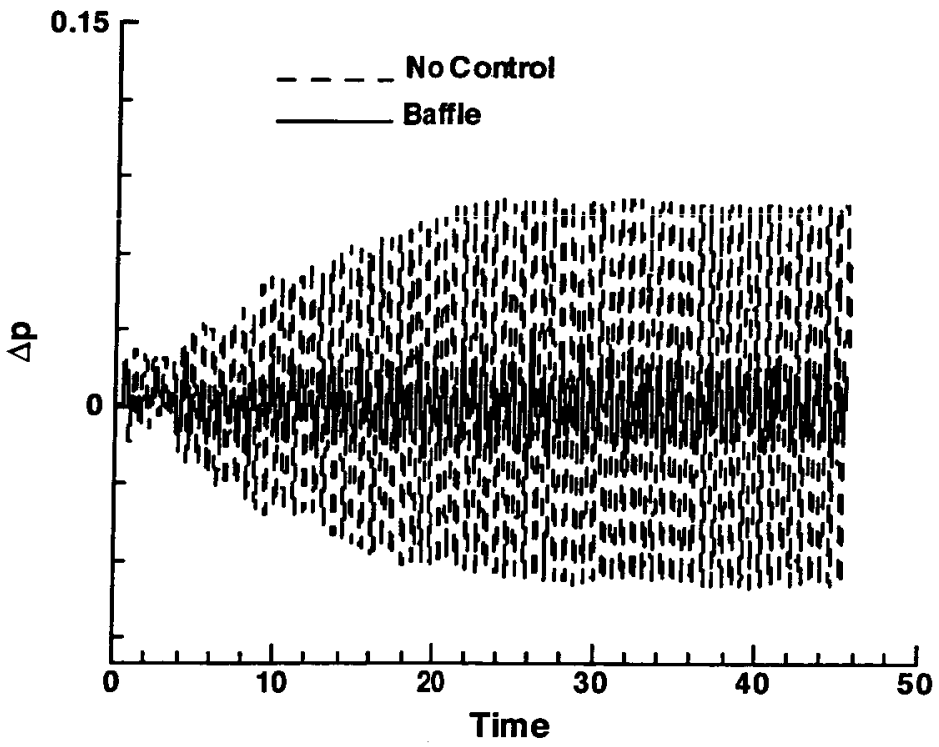

Figure 7: Effect of a radial baffle on the pressure oscillations in the chamber. 
Figure 8 shows the corresponding power spectra. This figure answers a critical question that can be asked based on Figure 7, which is: where did the excitation energy go? The answer based on Figure 8 is: the energy is redistributed on a broader frequency spectrum. The reason for this redistribution is that the excitation frequency, $1880 \mathrm{~Hz}$, is no-longer a fundamental mode of the chamber, therefore there is no resonance.

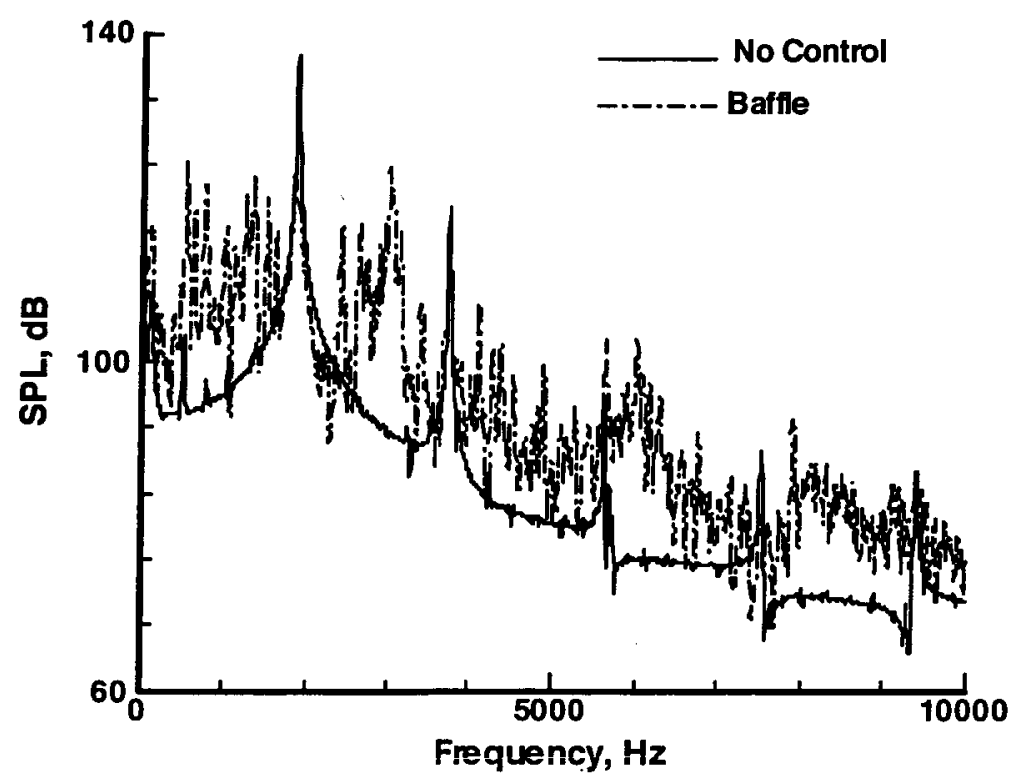

Figure 8: Power spectra of the pressure oscillations in the chamber with and without a baffle.

The effect of the baffle on the mode corresponding to the $512 \mathrm{~Hz}$ frequency is shown on Figs. 9(a)-(b). Figure 9(a) shows that after the excitation source is turned-off, Time $>30$, a weak decay of the pressure oscillations is obtained without using the baffle. When a baffle is used, Fig. 9(b), the decay rate is larger as indicated by the steeper exponential curve. Therefore, one can conclude that even though the mode corresponding to the $512 \mathrm{~Hz}$ frequency is not suppressed by the baffle, its damping is increased. This results is in agreement with the experimental measurements of Laudien et al. [13]. The overall sound pressure level in the chamber based on the root-mean-square (rms) pressure has also dropped from $170 \mathrm{~dB}$ to $156.5 \mathrm{~dB}$. 


\section{IV.2 Helmholtz Resonators}

Helmholtz resonators, known also as acoustic cavities, are very popular passive control devices. Their use is wide spread; from controlling instability waves to noise reduction devices such as iiners. A Heimholtz resonator is composed of an inlet of area $\mathbf{S}$ and length $\ell$
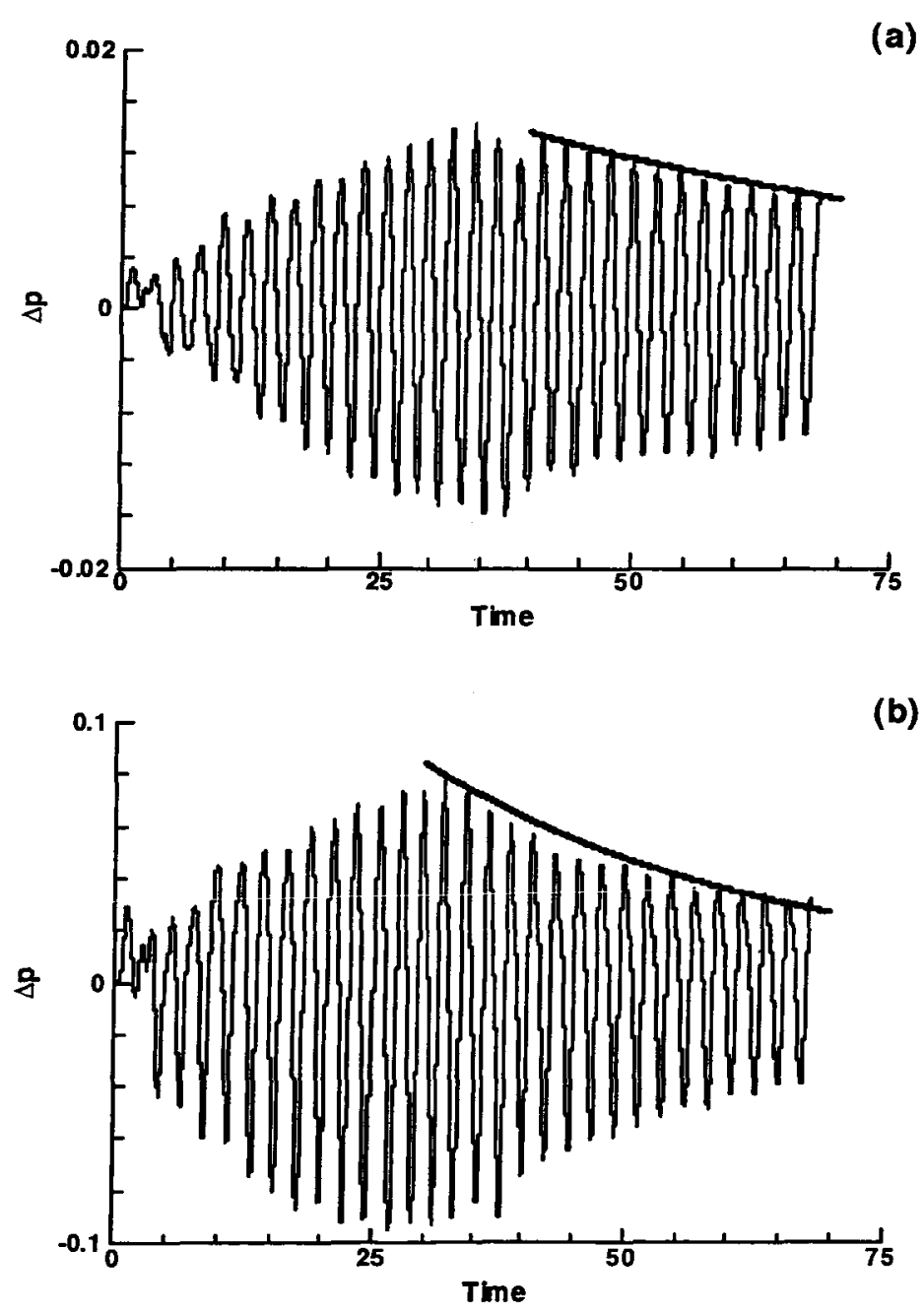

Figure 9: Time history of the chamber pressure along the top wall with the excitation source turned-on and then off (Time >30), (a) without a baffle, (b) with a baffle. 
followed by a cavity of volume $\mathbf{V}$, as shown in Fig. 10. For such a resonator, the resonar.frequency is given by

$$
f_{0}=\frac{c}{2 \pi} \sqrt{\frac{S}{V(\ell+\Delta \ell)}}
$$

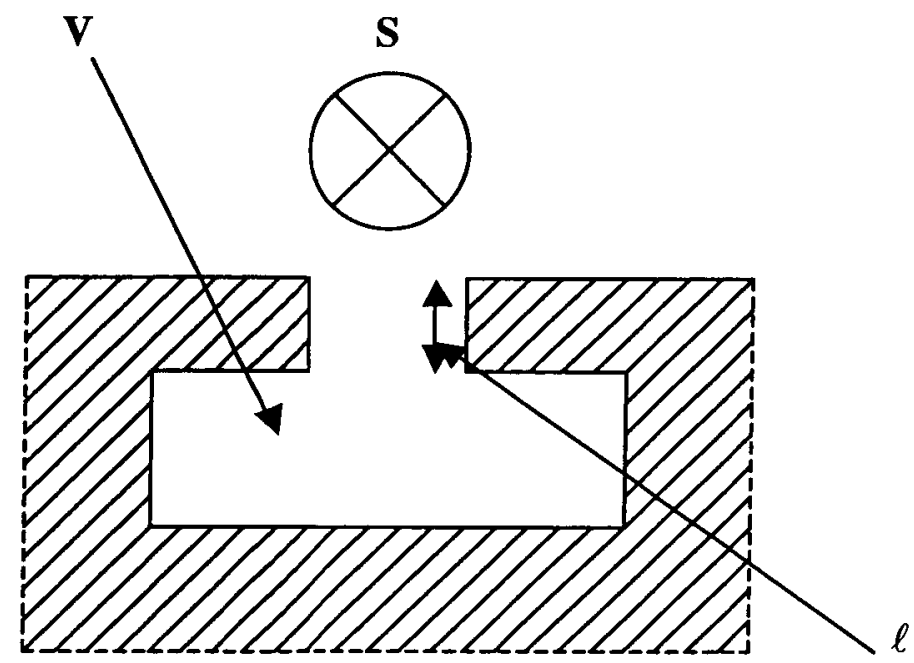

Figure 10: Typical geometry of a Helmholtz resonator.

where $\mathrm{c}$ is the speed of sound in the chamber and $\Delta \ell \approx 0.85 d$ with $d$ being the inlet diameter. The Helmholtz resonator used is tuned to a resonant frequency of $1880 \mathrm{~Hz}$, which corresponds to a chamber mode. When all the corners are sharp, Figure 11 shows that the presence of the resonator has reduced the pressure fluctuations in the chamber significantly. Similar to the baffle case, the power spectralshow the presence of more frequencies when the resonator is used, supporting the argument that energy has been diverted from the fundamental to other frequencies. The overall sound pressure level in the chamber based on the rms-pressure has also dropped from $170 \mathrm{~dB}$ to $154 \mathrm{~dB}$ ( $2.5 \mathrm{dBs}$ better than the baffle). 


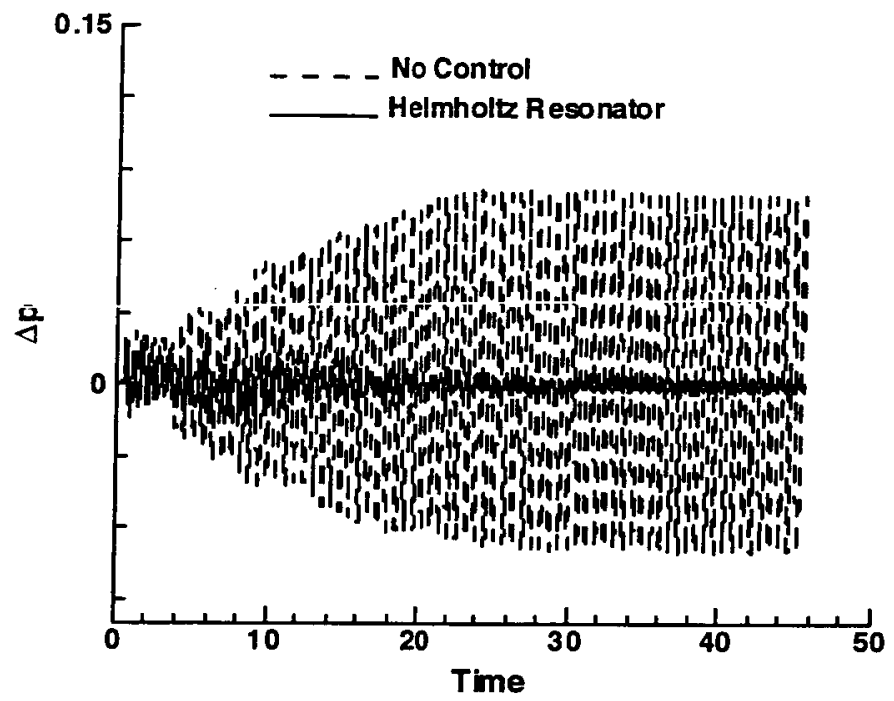

Figure 11: Effect of a Helmholtz resonator on the pressure oscillations in the combustion chamber.

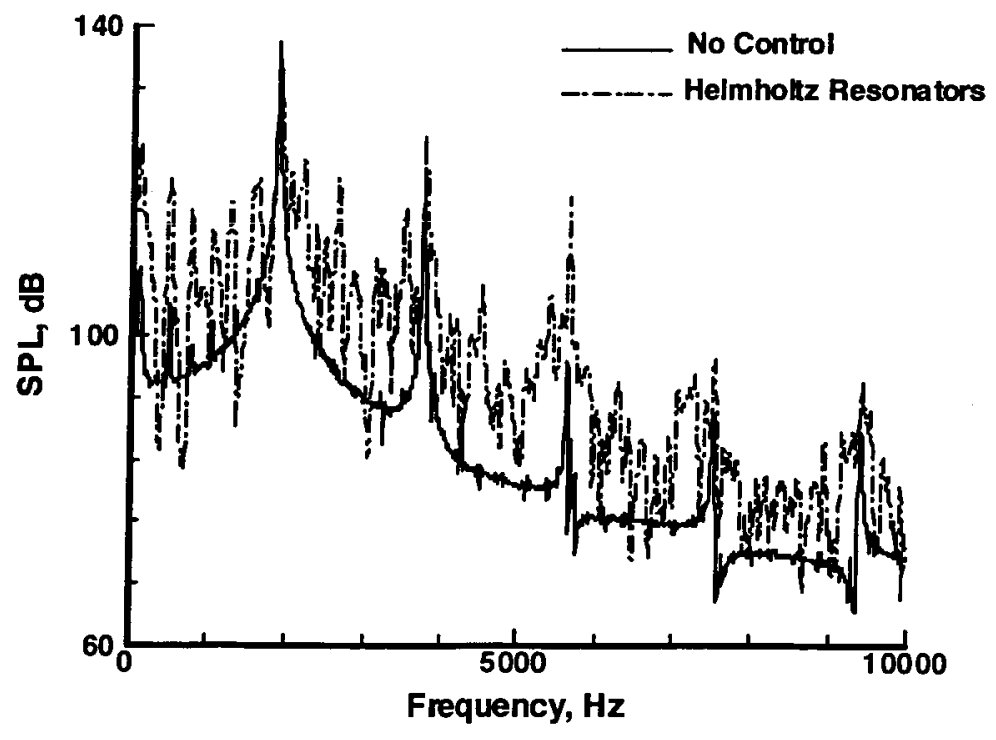

Figure 12: Power spectra of the pressure oscillations in the chamber with and without a resonator. 
The effect of smoothing the various corners of the resonator on the overall sound pressure level in the chamber is shown on Figure 13. The figure shows that the best result is obtained when both inlet corners (referred to as throat corners on the Figure) are smoothed. Smoothing the other interior resonators corners did not achieve any additional improvement, as shown by the figure. Notice that "iniet corner" on the figure refers to the corner facing the combustion chamber.

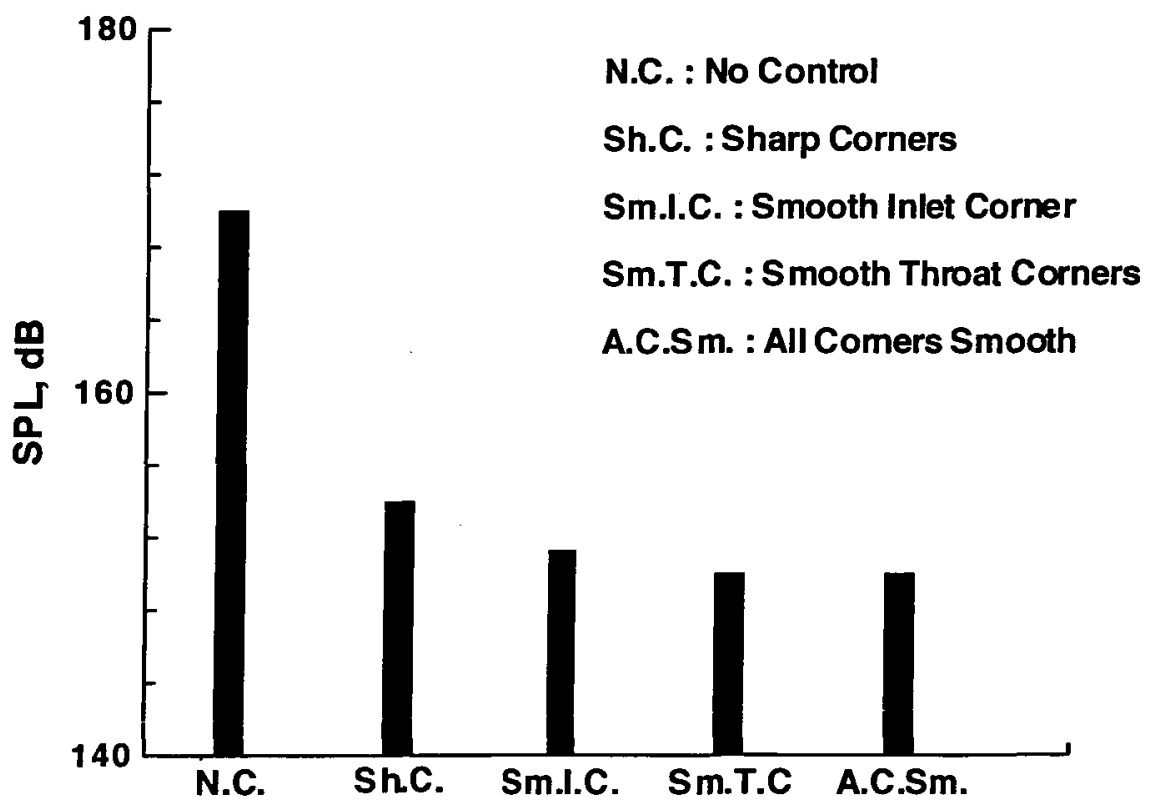

Figure 13: Effect of smoothing the resonator corners on the overall sound pressure level.

According to the experimental results of Laudien et al. [13], the resonator becomes less effective at higher noise levels in the chamber. This result is confirmed by our computations, which show less-drop in dB-level, Figure 14, even with the best configuration of Fig. 13. 


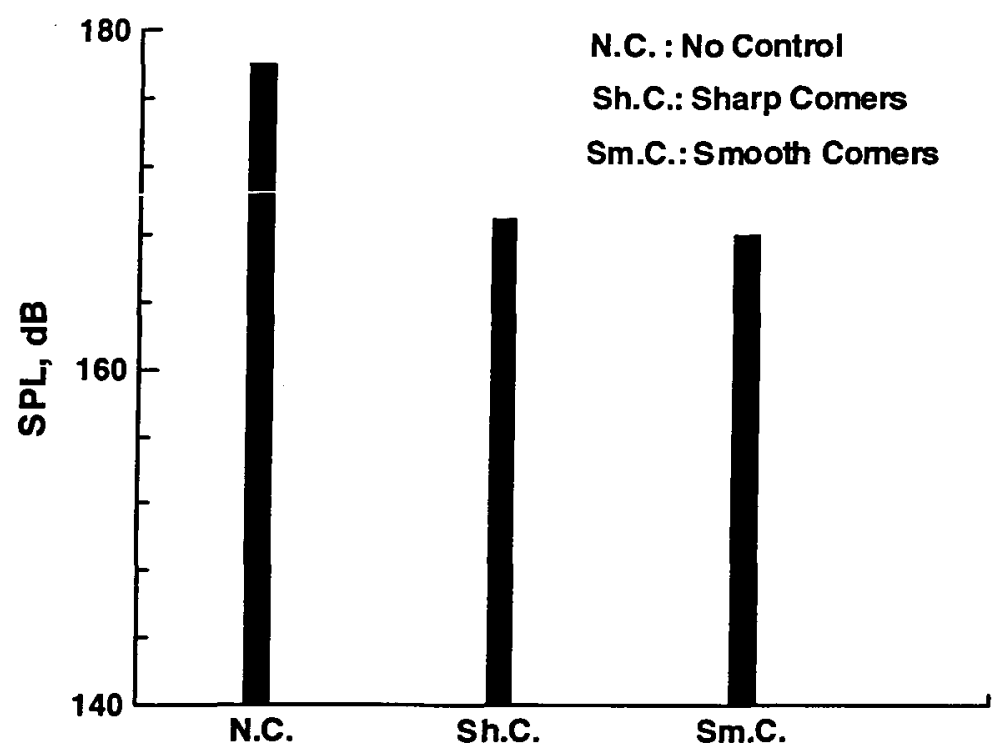

Figure 14: Effect of a Helmholtz resonator on the overall sound pressure level of a combustion chamber at high noise levels.

The problem in designing a Helmholtz resonator is the required knowledge of the tuning or resonant frequency. Therefore, one question we needed to answer is how effective a resonator is when the design frequency is slightly off? This is of practical importance since in an actual combustion experiment the frequency to be controlled is not known accurately. To answer this question, a resonator tuned to a frequency of $1800 \mathrm{~Hz}$ was used to control a mode of frequency $1880 \mathrm{~Hz}$. Figure 15 shows the overall sound pressure level in the chamber with an "On-Design" and an "Off-Design" resonator. The "Off-Design" resonator achieves 13.5 $\mathrm{dB}$ less reduction than the "On-Design" one. This is significant since the "Off-Design" frequency is only $80 \mathrm{~Hz}$ off the design value. 


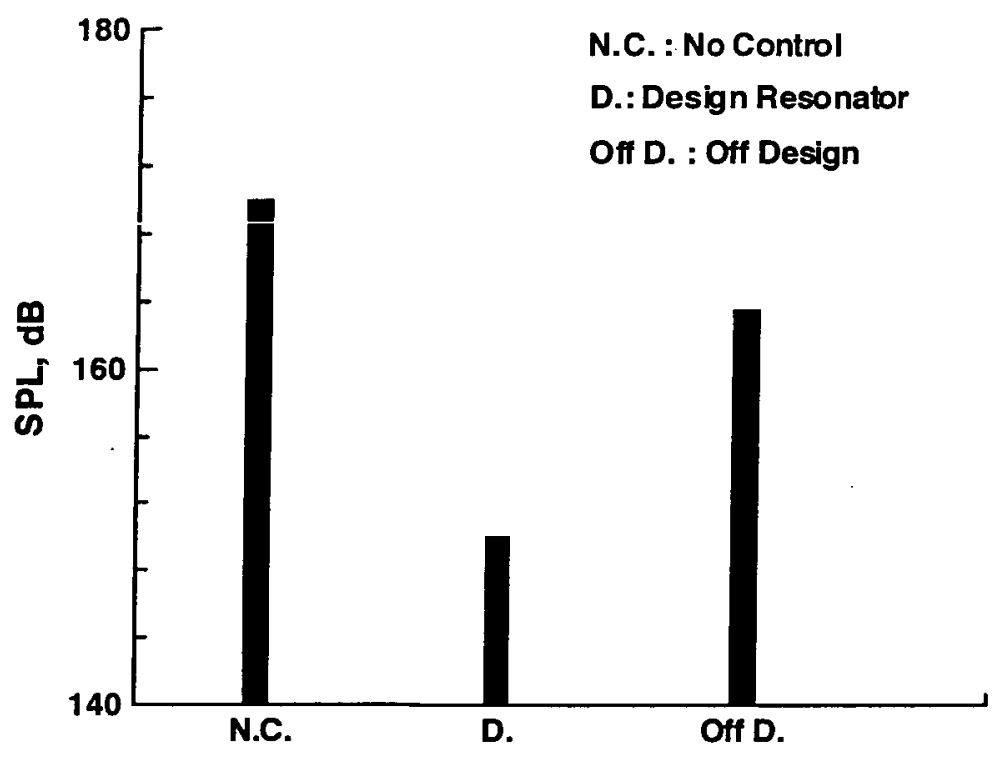

Figure 15: Effect of resonator design frequency on the overall sound pressure level in the combustion chamber.

\section{IV.3 Quarter-Waves}

Another passive control device commonly used is a quarter-wave. It is somewhat similar to a Helmholtz resonator except for the absence of the cavity part, Figure 16. The resonant frequency of a quarter-wave is given by

$$
f_{0}=\frac{c}{4(L+\Delta \ell)}
$$

with $\mathrm{c}$ being the speed of sound and $\Delta \ell \approx 0.85 d$ with $d$ the quarter-wave diameter. 


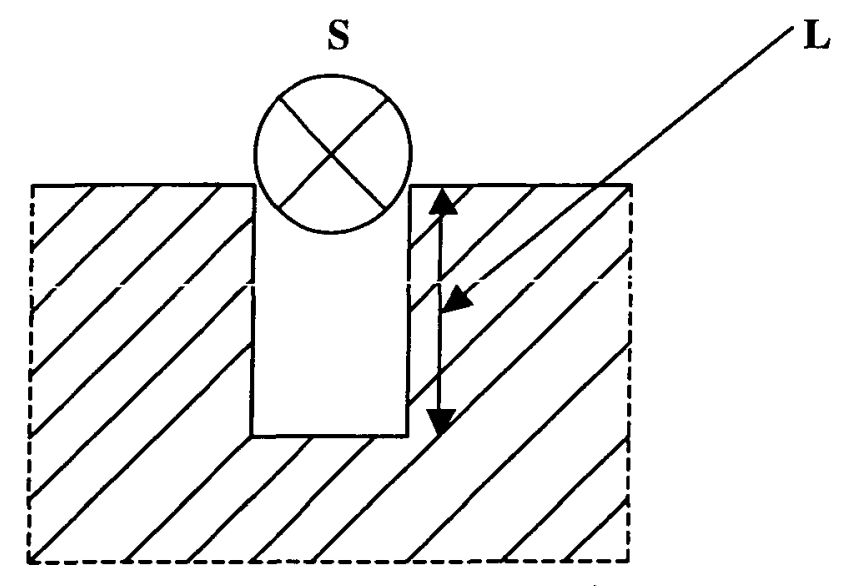

Figure 16: Typical geometry of a quarter-wave.

In practice, several shapes of quarter-waves are used. In this study two of these shapes will be investigated; an L-shaped quarter-wave and a straight quarter-wave. In addition, the effect of smoothing the corners will be analyzed. Figure 17 shows the time history of the chamber pressure with and without a tuned quarter-wave. A significant reduction in pressure oscillations is achieved when using a quarter-wave. Figure 18 shows the corresponding power spectra. Similar to the baffle and resonator, the quarter-wave effect is to detune the

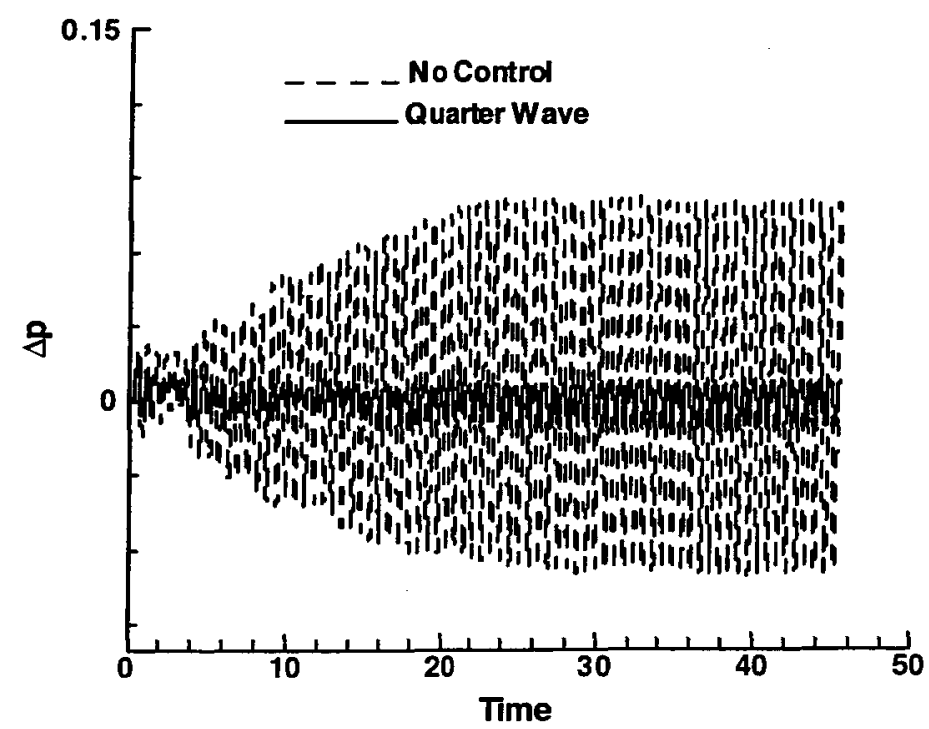

Figure 17: Effect of a quarter-wave on the pressure oscillations in the combustion chamber. 


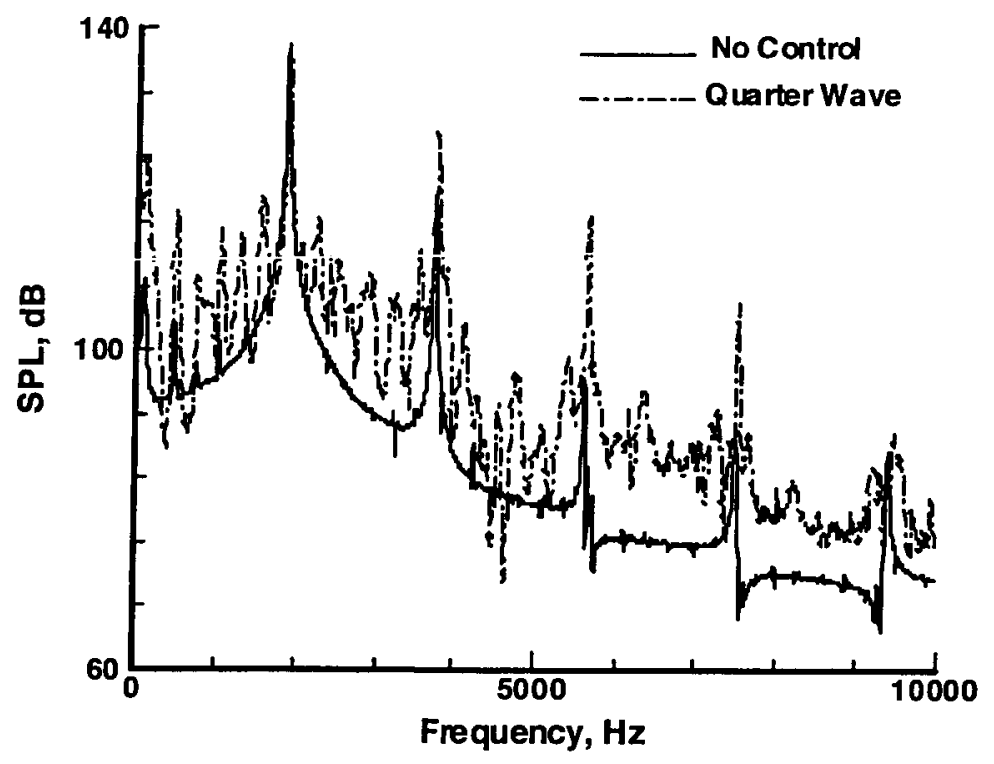

Figure 18: Power spectra of the pressure oscillations in the chamber with and without a quarter-wave.

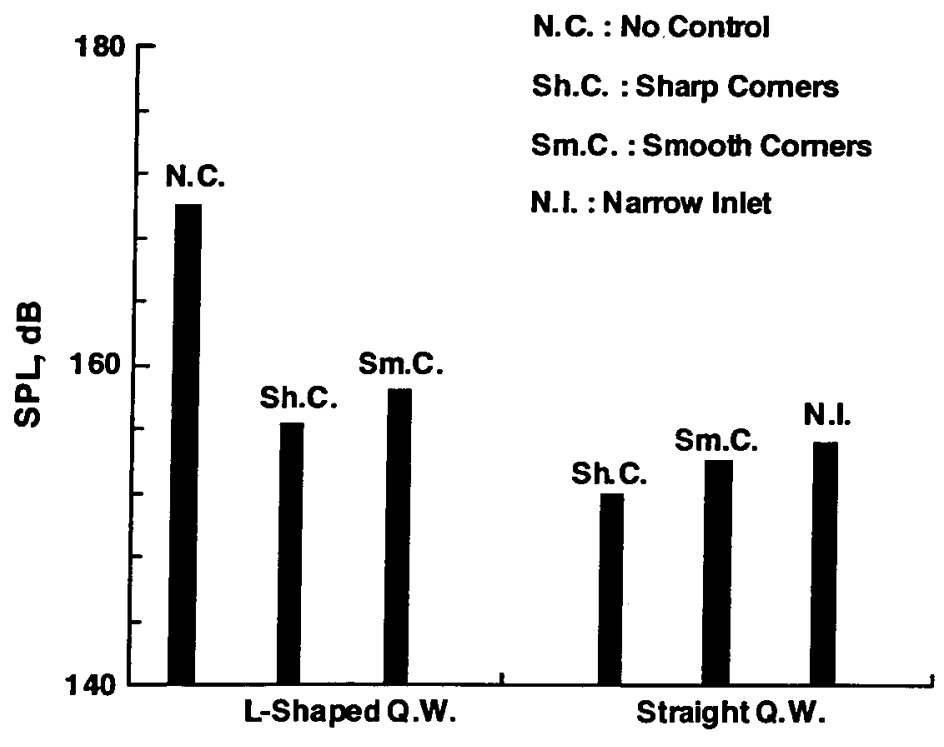

Figure 19: Effect of L-shaped and Straight quarter-waves with different corner geometries on the overall sound pressure level in the chamber. 
chamber and spread the energy to other frequencies. A comparison of an $\mathrm{L}$ shaped quarterwave to a straight one with various corner geometries is shown on Figure 19. For all corner geometries, the straight quarter-wave achieves more reduction in SPL than the L-shaped one. The figure also shows that smoothing the inlet corners has an adverse effect on the overall SPL in the chamber for both quarter-wave geometries. For the straight quarter-wave, a narrow inlet resulted in a higher SPL, as expected.

Figure 20 shows a comparison of overall SPL in the combustion chamber for all the passive devices tested in this study. The figure shows that the Helmholtz resonator with a smooth inlet gives the best results.

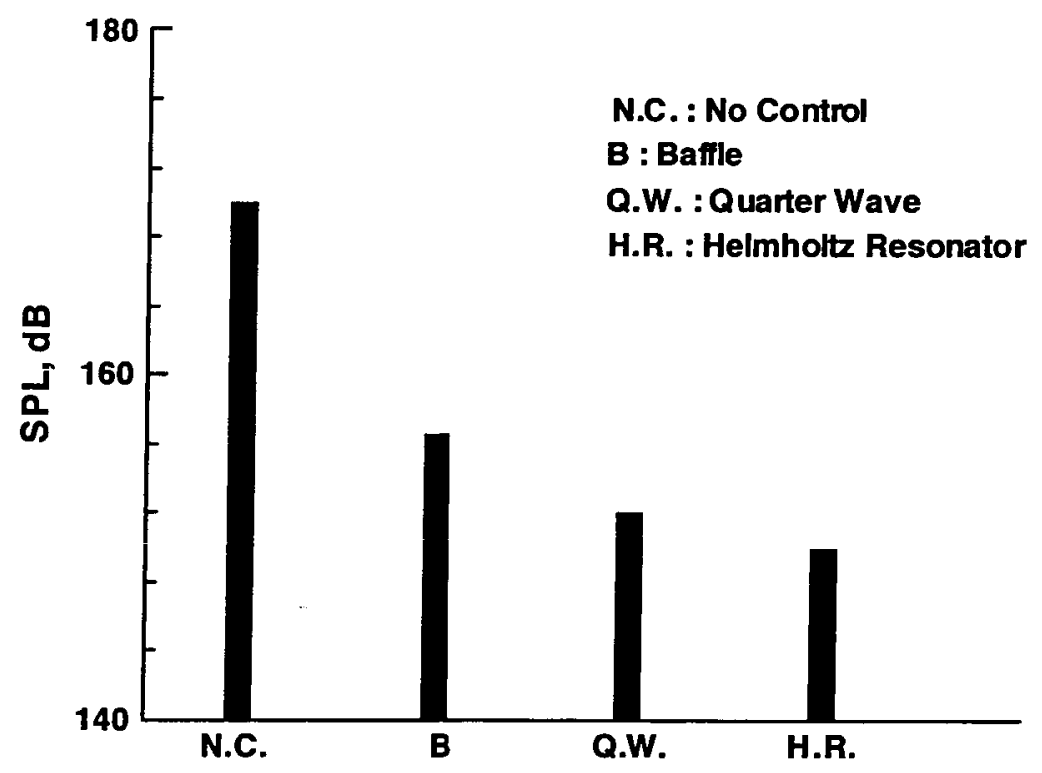

Figure 20: Comparison of overall sound pressure level in the chamber obtained with the various passive devices.

Figures 21-24 show the instantaneous pressure contours in the chamber obtained with the various passive devices. A different pattern can be observed for each device. 


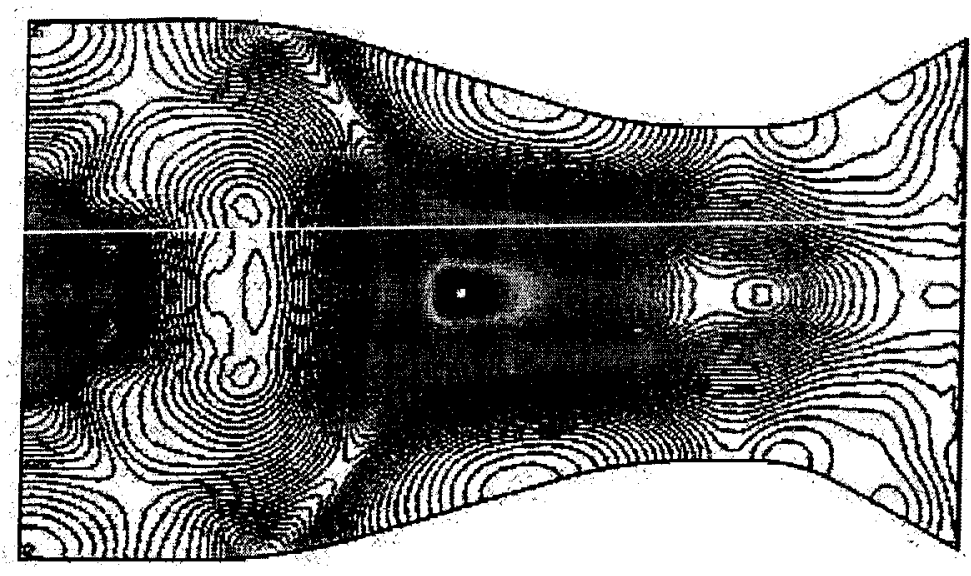

Figure 21: Instantaneous pressure contours in the combustion chamber obtained without a passive device.

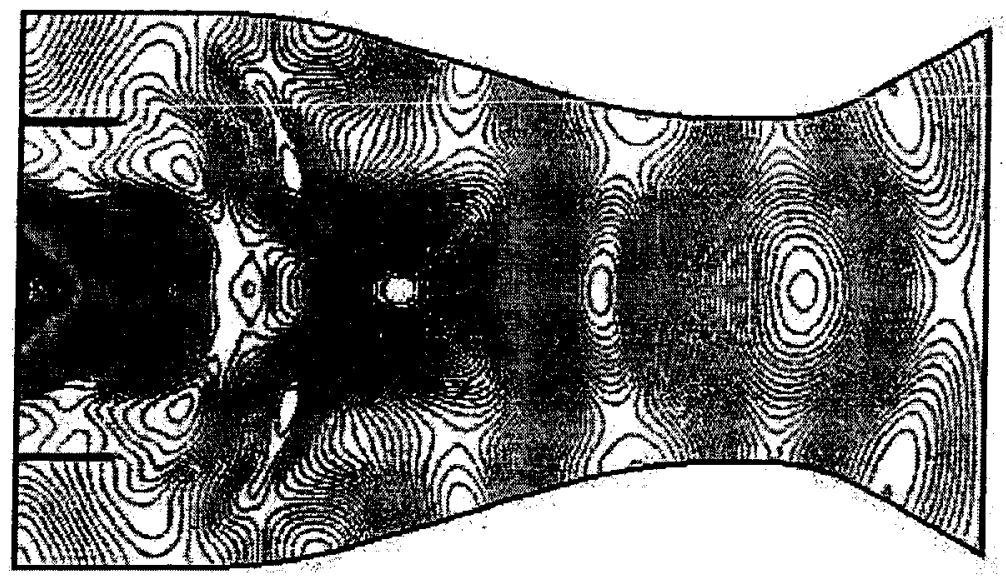

Figure 22: Instantaneous pressure contours in the combustion chamber obtained with a baffle. 


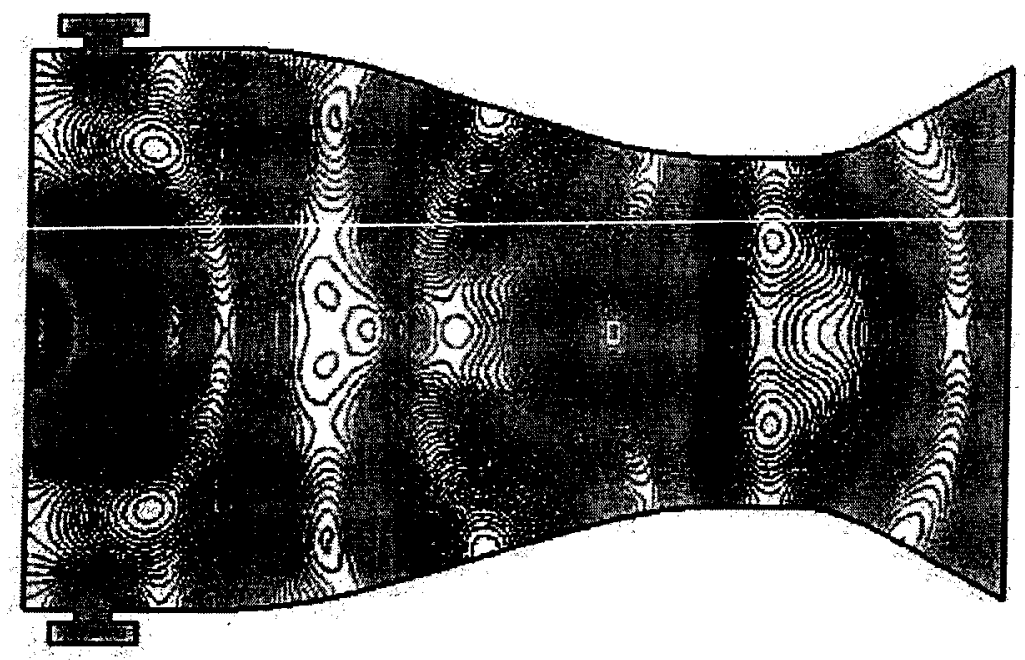

Figure 23: Instantaneous pressure contours in the combustion chamber obtained with a Helmholtz resonator.

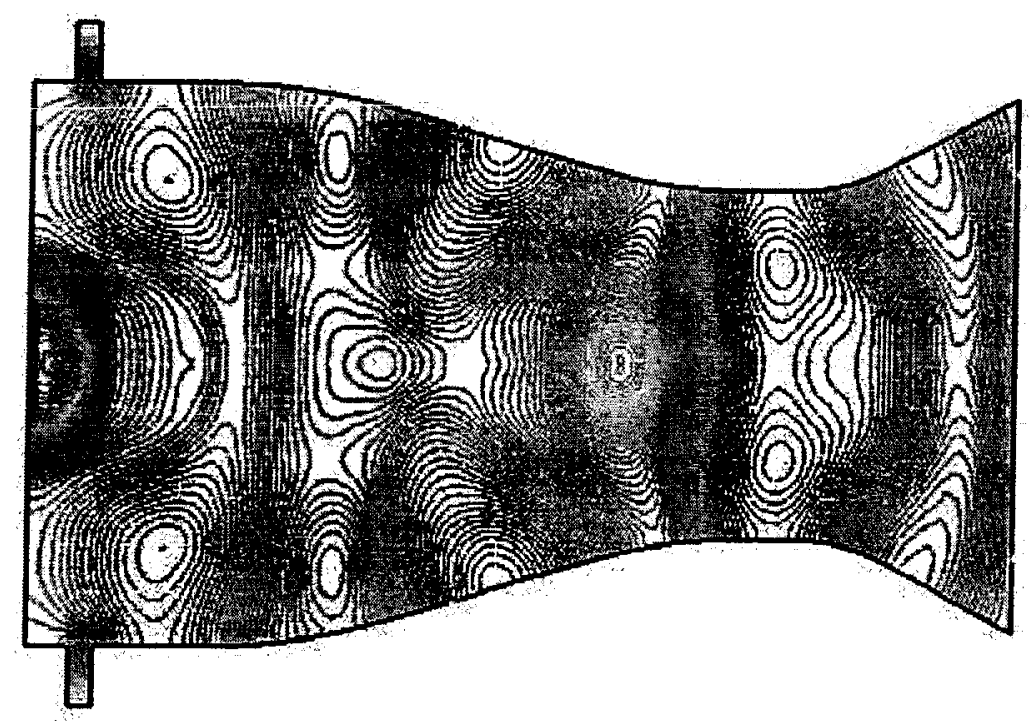

Figure 24: Instantaneous pressure contours in the combustion chamber obtained with a quarter-wave. 


\section{Concluding Remarks}

Numerical experiments have been carried-out to study the effectiveness of various passive devices to control combustion instabilities. The results show that, for the given mode studied, a Helmholtz resonator with a smooth inlet achieves the best control results. However, at high sound pressure levels in the chamber, the resonator becomes less effective. For the case of a quarter-wave, it is found that a straight shape performs better than an L-shaped quarter-wave. In addition, smoothing the corners of a quarter-wave inlet may have an adverse effect on the overall sound pressure level in the chamber. The computational results presented here are in good qualitative agreement with the experimental measurements of Laudien et al. [13].

\section{Acknowledgements}

This work was supported by a summer faculty fellowship from NASA Marshall Space Flight Center with Tom Nesman as the technical monitor. 


\section{References}

[1] Yang, V., and Anderson, W.E., "Liquid Rocket Engine Combustion Instability", Progress in Aeronautics and Astronautics, Vol. 169, Editor-in-Chief Paul Zarchan, 1995.

[2] "Liquid Propellant Rocket Combustion Instability", NASA SP-194, Edited by Dãviu T. Harrje, 1972.

[3] Giammar, R.D., and Putnam, A.A., "Combustion Roar of Premix Burners, Singly and in Pairs," Combustion and Flame, Vol, 18, 1972, pp. 435-438.

[4] Karchmer, A.M., "Identification and Measurement of Combustion Noise from a Turbofan Engine Using Correlation and Coherence Techniques," NASA TM-73747, 1977.

[5] Rayleigh, J.W.S., "The Explanation of Certain Acoustical Phenomena," Nature, Vol. 18,1878, p. 319.

[6] Bloxidge, G.J., Dowling, A.P., Hooper, N., and Langhorne, P.J., "Active Control of Reheat Buzz," AIAA Journal, Vol. 26, 1988, pp. 783-790.

[7] Abouseif, G.E., Keklak, J.A., and Toong, T.Y., "Ramjet Rumble: The Low Frequency Instability Mechanism in Coaxial Dump Combustors," Combustion Science and Technology, Vol. 36, 1984, pp. 83-108.

[8] Yang, V. and Culick, F.E.C., "Linear Theory of Pressure Oscillations in LiquidFueled Ramjet Engines," AIAA Paper 83-0574, 1983.

[9] McIntosh, A.C., "The Effect of Upstream Acoustic Forcing and Feedback on the Stability and Resonance of Anchored Flames," Combustion Science and Technology, Vol. 49, 1986, pp. 143-167. 
[10] Shyy, W., Thakur, S., Udayk umar, H.S., "A High Accuracy Sequential Solver for Simulation and Active Control of Longitudinal Combustion Instability," Computing Systems in Engineering, Vol. 4, No. 1, 1993, pp. 27-41.

[11] Prasad, K., "Interaction of Presure Perturbations with Premixed Flames", Combustion and Flames, Vol. 97, pp. 713-200, 1994.

[12] Frendi, A., "On the Role of Acoustic Coupling on Combustion Instabilities", AIAA2002-3181, Hilton Head, South Carolina, 2003.

[13] Laudien, E., Pongratz, R., Pierro, R., and Preclik, D., "Experimental Procedure Aiding the Design of Acoustic Cavities", Liquid Rocket Engine Combustion Instability, edited by Vigor Yang and William Anderson, Progress in Astronautics and Aeronautics, Vol. 169, pp.377-399, 1995.

[14] Canabal, F., and Frendi, A, "A Finite Element Based Flux Correction Scheme for the Euler Equations", AIAA-2003-3121.

[15] Canabal, F., "Prediction and Suppression of Ignition Overpressure", $\mathrm{PhD}$ Thesis, University of Alabama in Huntsville, 2004. 